\section{Functional rescue of experimental ischemic optic neuropathy with $\alpha \mathrm{B}$-crystallin}

S Pangratz-Fuehrer ${ }^{1}$, K Kaur $^{1}$, SS Ousman², L Steinman $^{2}$ and YJ Liao ${ }^{1}$
Purpose Anterior ischemic optic neuropathy (AION) is an important cause of acute vision loss in adults, and there is no effective treatment. We studied early changes following experimental AION and tested the benefit of a potential treatment.

Materials and Methods We induced experimental AION in adult mice and tested the effects of short-term (daily for 3 days) and long-term (every other day for 3 weeks) $\alpha \mathrm{B}$-crystallin $(\alpha \mathrm{BC})$ treatment using histological and serial intracranial flash visual evoked potential recordings.

Results One day after experimental AION, there was swelling at the optic nerve (ON) head and increased expression of $\alpha \mathrm{BC}$, a small heat shock protein important in ischemia and inflammation. This upregulation coincided with microglial and astrocytic activation. Our hypothesis was that $\alpha \mathrm{BC}$ may be part of the endogenous protective mechanism against injury, thus we tested the effects of $\alpha \mathrm{BC}$ on experimental AION. Daily intraveneous or intravitreal $\alpha \mathrm{BC}$ injections did not improve visual evoked potential amplitude or latency at days 1-2. However, $\alpha \mathrm{BC}$ treatment decreased swelling and dampened the microglial and astrocytic activation on day 3 . Longer treatment with intravenous $\alpha B C$ led to acceleration of visual evoked potential latency over 3 weeks, without improving amplitude.

This latency acceleration did not correlate with increased retinal ganglion cell survival but did correlate with complete rescue of the $\mathrm{ON}$ oligodendrocytes, which are important for myelination.

Conclusions We identified $\alpha \mathrm{BC}$ as an early marker following experimental AION. Treatment with $\alpha \mathrm{BC}$ enhanced this endogenous, post-ischemic response by decreasing microglial activation and promoting $\mathrm{ON}$ oligodendrocyte survival. Eye (2011) 25, 809-817; doi:10.1038/eye.2011.42; published online 8 April 2011

Keywords: anterior ischemic optic neuropathy; $\alpha \mathrm{B}$-crystallin; visual evoked potential; retinal ganglion cell; heat shock protein

\section{Introduction}

Anterior ischemic optic neuropathy (AION) is the most common acute optic neuropathy in patients over 50-years-old and typically leads to permanent vision loss. ${ }^{1-2}$ Although experts agree that non-arteritic AION results from ischemia of the optic nerve (ON) head, ${ }^{1,3}$ there is still significant controversy regarding the mechanism of disease $e^{4}$ and there is currently no effective treatment. Photochemical thrombosis animal models of AION have been instrumental in elucidating the events following $\mathrm{ON}$ head ischemia. ${ }^{5-9}$ In experimental AION, there is early glial and axonal dysfunction, with $\mathrm{ON}$ oligodendrocyte apoptosis occurring by day 6 and progressive nerve demyelination within 2 weeks. ${ }^{5,8,9}$ Retinal ganglion cell (RGC) loss

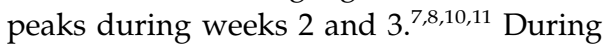
these 3 weeks of critical period for treatment considerations, there are important signs of self repair, such as the upregulation of retinal heat shock proteins HSP 70, 84, and 86. ${ }^{5,8}$ Heat shock proteins act as molecular chaperones and are thought to have important roles in optic neuropathies, retinal diseases, and central nervous system inflammatory and neurodegenerative diseases. ${ }^{12-16}$

In this study, we used a mouse model for AION via photochemical thrombosis ${ }^{5-8,10,11,17}$ to examine the early changes following $\mathrm{ON}$ head ischemia. We then assessed the effectiveness of a small heat shock protein named $\alpha \mathrm{B}$-crystallin
${ }^{1}$ Department of Ophthalmology, Stanford University School of Medicine, Stanford, CA, USA

${ }^{2}$ Department of Neurology, Stanford University School of Medicine, Stanford, CA, USA

Correspondence: YJ Liao, Department of Ophthalmology, Byers Eye Institute at Stanford, 2452 Watson Court, Palo Alto, CA 94303, USA

Tel: + 1650723 6995;

Fax: + 16507256619 .

E-mail: yjliao@

stanford.edu

Received: 16 November 2010

Accepted in revised form: 27 January 2011 Published online: 8 April 2011

This work has been presented at NANOS 2009, SFN 2009, and ARVO 2009 
$(\alpha \mathrm{BC})$ in the treatment of experimental AION, using functional and histological approaches.

\section{Materials and methods}

\section{Animals, care, and sedation}

All animal care and experiments were carried out in accordance with the ARVO guideline for the Use of Animals in Ophthalmic and Vision Research and the Stanford Administrative Panel on Laboratory Animal Care. Adult wild-type 129S2/Sv mice were purchased (Charles River, Wilmington, MA, USA; Taconic Farm, Hudson, NY, USA) and housed in a 12-h light/12-h dark cycle with food and water ad libitum. All procedures were performed under sedation, achieved with ketamine $50-100 \mathrm{mg} / \mathrm{kg}$, xylazine $2-5 \mathrm{mg} / \mathrm{kg}$, and buprenorphine $0.05 \mathrm{mg} / \mathrm{kg}$.

\section{Experimental AION}

Our model of experimental AION is a modification of previously published studies. ${ }^{5-8,10,11,17}$ After rose bengal (1.25 mM in PBS, $2 \mu$ l per g body weight) injection via tail vein, the $\mathrm{ON}$ head was exposed to 20 light spots (frequency doubled Nd:YAG laser, Pascal, OptiMedica, Santa Clara, CA, USA), each with $100 \mu \mathrm{m}$ diameter (equivalent to $39 \mu \mathrm{m}$ on the nerve), at a power of $35 \mathrm{~mW}$ and duration of $500 \mathrm{~ms}$. We delivered small laser spots at low power and shorter duration circumferentially around the $\mathrm{ON}$ head in order to focus more precisely while covering the entire $\mathrm{ON}$ head. The degree and extent of whitening due to ischemia ${ }^{18}$ are similar to that of the Bernstein model in our direct comparison. As reported previously, ${ }^{5,8}$ application of the laser in the absence of rose bengal did not cause significant ischemia.

\section{Western blotting}

Protein was prepared from the $\mathrm{ON}$ head and sensory retina of three pairs of eyes from adult mice 1 day post-AION per routine protocol in the presence of protease inhibitors. Protein concentrations were determined using micro BCA kit (Pierce, Rockford, IL, USA). Equal amount of protein per lane was loaded onto a 10\% mini-SDS-PAGE gel (Invitrogen, Carlsbad, CA, USA) and run as per the recommended protocol. Western blotting was carried out with anti- $\alpha \mathrm{BC}$ rabbit polyclonal antibody ( $1: 200$ dilution, Abcam, Cambridge, MA, USA) and anti-class III $\beta$-tubulin monoclonal antibody (1:500 dilution, Abcam) and developed per the ECL protocol (Pierce). Four experiments for each antibody were scanned under equal conditions and quantified with Image J (http:/ /imagej.nih.gov/). Student's paired $t$-test was used for statistical analysis, and all error bars are in SEM.

\section{Immunohistochemistry and morphometric analyses}

At each time point, we performed intracardiac perfusion (India ink for angiogram, 4\% paraformaldehyde in PBS for fixation) and whole mount neuroretinal dissection. For immunofluorescence microscopy, primary antibody with anti-Iba-1 rabbit polyclonal antibody (1:500-1000 dilutions, WAKO, Richmond, VA, USA) or anti-glial fibrillary acidic protein (GFAP) monoclonal antibody (1:500-1000 dilutions, Sigma, St Louis, MO, USA) and goat anti-rabbit or anti-mouse IgG secondary antibodies ( $1: 200-400$ dilutions, Invitrogen) were used. The retinas were mounted with Vectashield containing DAPI (Vector Labs, Burlingame, CA, USA). For paraffin-embedded or frozen horizontal sections, the eyes and ONs were dissected, and $10 \mu \mathrm{m}$ horizontal sections were prepared and $\mathrm{H}$ and $\mathrm{E}$ stained. All morphometric analysis was performed under masked condition so the identity of the data was not known. A circular area (300 pixel radius) centered at the $\mathrm{ON}$ head was quantified with Image J. Following background subtraction, regions of interest were multiplied by the intensity to calculate the intensity* pixel $^{2}$. For each pair of eyes, the AION eye was normalized to the unlasered eye, which served as control for every step of the experiment. Statistical significance between the lasered and unlasered eyes was determined using paired Student's $t$-test and between saline and $\alpha \mathrm{BC}$-treated groups, unpaired $t$-test.

\section{$\alpha B C$ treatment}

For intravenous treatment, $10 \mu \mathrm{g}$ of recombinant $\alpha \mathrm{BC}$ (U.S. Biological, Swampscott, MA, USA) vs saline alone (final volume $100 \mu \mathrm{l}$ ) was injected into the tail vein under masked conditions within $5 \mathrm{~h}$ of AION. For short-term treatment, the animals were injected once per day for 3 days. For longer treatments, the animals were injected every other day for 3 weeks. Animals in the experimental and control groups received same number of injections. To see if intravitreal injection of $\alpha B C$ led to additional benefit compared with intravenous injections alone, we performed intravitreal injection of $2 \mu \mathrm{g} \alpha \mathrm{BC}$ or saline (final volume $1 \mu \mathrm{l}$ ) given to each eye within $5 \mathrm{~h}$ of $\mathrm{AION}$ in addition to intravenous injections.

\section{Intracranial electrode implantation}

Small burr holes were drilled in the skull overlying the visual cortex on each side (2.5-3 mm parasagittal and $2-3 \mathrm{~mm}$ anterior to the lambdoid suture ${ }^{19,20}$ ) for placement of small stainless steel screw electrodes (JI Morris, Southbridge, MA, USA). Dental cement secured the electrodes over the skull, with several sutures to close the scalp. Implanted mice behaved like unimplanted mice, with normal response to stimuli, locomotion and weight gain over months. 


\section{Visual evoked potential recording}

We used serial intracranial photopic flash visual evoked potential (fVEP) recordings to monitor ON function, assuming no changes elsewhere. Recordings were conducted under standardized conditions (sedation, body temperature, circadian rhythm, hydration status). A reference electrode was inserted near the nose, and a ground electrode, near the tail. Impedance was monitored before each trial. In the Ganzfeld dome (Espion $E^{2}$, Diagnosys, Westford, MA, USA), implanted mice (10 mice per group, 4-5 months old) were exposed to white light flashes at $2 \mathrm{~Hz}$ in background luminance of $20 \mathrm{~cd} / \mathrm{m}^{2}$. Data were acquired at $1 \mathrm{kHz}$ and band pass filtered at $0.5-100 \mathrm{~Hz}$. Each fVEP response trial was the average of 50 traces, with multiple trials recorded per luminance intensities $\left(0.001-20 \mathrm{~cd} \mathrm{~s} / \mathrm{m}^{2}\right)$ per day. A fVEP of relatively large amplitude is shown in Figure $3 a$ to illustrate responses over a large range of luminance intensities. There were no fVEP responses when both eyes were occluded. The recordings were conducted while masked to the treatment given.

The amplitude and latency of $\mathrm{N}_{1}$, the dominant fVEP waveform, were averaged from 30-50 trials (minimum of three trials/mouse/time point) from six animals per treatment group, where good recording was performed in every time point. Data analysis was restricted to luminance intensities between $2.15-20 \mathrm{~cd} \mathrm{~s} / \mathrm{m}^{2}$. Raw amplitude was calculated as difference between baseline and $\mathrm{N}_{1}$ peak ranged between $3-50 \mu \mathrm{V}$. The latency was calculated as time to $\mathrm{N}_{1}$ peak from stimulus onset and ranged between $30-80 \mathrm{~ms}$. Although latencies for $\mathrm{N}_{1}$ were extremely stable across several examinations in each mouse, their raw amplitudes showed more variability. Data were analyzed with Excel and Origin, Grubbs' test was used to eliminate outliers ( $0-3$ per data point), and statistical differences were determined using Student's $t$-test and one-way ANOVA.

\section{RGC quantification}

$\mathrm{H}$ and $\mathrm{E}$ stained horizontal sections of the posterior pole were photographed with a $\times 40$ objective. Under masked conditions, the number of cells in the RGC layer per high power field was quantified using Image J. Following color deconvolution, the entire RGC layer was counted with nucleus counter, using a size threshold of 500 pixels, and then visually confirmed. Statistical significance was determined using unpaired Student's $t$-test.

\section{ON oligodendrocyte quantification}

We stained for oligodendrocytes with anti-CC1 mAb antibody (Calbiochem, Gibbstown, NJ, USA), goat anti-mouse secondary antibody, and mounted with
DAPI-containing Vectorshield medium (Vector Labs). We photographed the first $500 \mu \mathrm{m}$ of the myelinated ONs and used image $\mathrm{J}$ to quantify the number of cells that were CC1-negative and DAPI-positive, which represented oligodendrocytes. $\mathrm{CC} 1$ antibody staining did not overlap with that of GFAP expression in astrocytes. Statistical significance was determined using unpaired Student's $t$-test.

\section{Statement of ethics}

We certify that all applicable institutional and governmental regulations concerning the ethical use of animals were followed during this research.

\section{Results}

\section{Early post-AION upregulation of $\alpha B C$}

Immediately following experimental AION, there was loss of peripapillary microvasculature, swelling, and leakage at the ON head (Figure 1a). At day-1 following $\mathrm{AION}$, western blot analysis (Figure $1 \mathrm{~b}-\mathrm{c}$ ) revealed an upregulation of $\alpha \mathrm{BC}$ protein in the $\mathrm{ON}$ head and neuroretina compared with control eyes $(n=4$ experiments, $P<0.03$ ), but no significant change in the expression of class-III $\beta$-tubulin, a major component of the neuronal microtubule that is highly expressed in RGCs. Consistent with the idea that $\alpha \mathrm{BC}$ is important in inflammation, ${ }^{15,21}$ we found that on post-ischemia day 3 , there was increased ON head Iba-1 staining (data not shown, $n=5$, see Figure 2), which suggested microglial activation, and elevated GFAP expression (data not shown, $n=5$, see Figure 2), which correlated with astrocytic activation. These findings resembled changes after laser-induced photochemical thrombosis in the retina $^{22}$ and axonal ischemia near the retina-ON junction $^{23}$ and the brain. ${ }^{24}$ Our data indicated that $\alpha \mathrm{BC}$ was an early marker following experimental AION, which may serve as part of the endogenous protective mechanism.

\section{Short-term $\propto B C$ treatment}

To test this hypothesis, we performed short-term and long-term $\alpha \mathrm{BC}$ treatment following experimental AION in adult mice. For short-term treatment, animals received daily intravenous injections of $\alpha \mathrm{BC}$ ( $10 \mu \mathrm{g}$ per injection) vs phosphate-buffered saline (PBS) alone for 3 days, and we performed immunohistochemistry and analyzed whole mount retina for $\mathrm{ON}$ head staining with Iba-1 antibody for microglia and with anti-GFAP antibody for astrocytes. In the PBS-treated group, like in non-treated eyes, the Iba-1 expression following AION was significantly increased compared with that of the control 


\section{a}

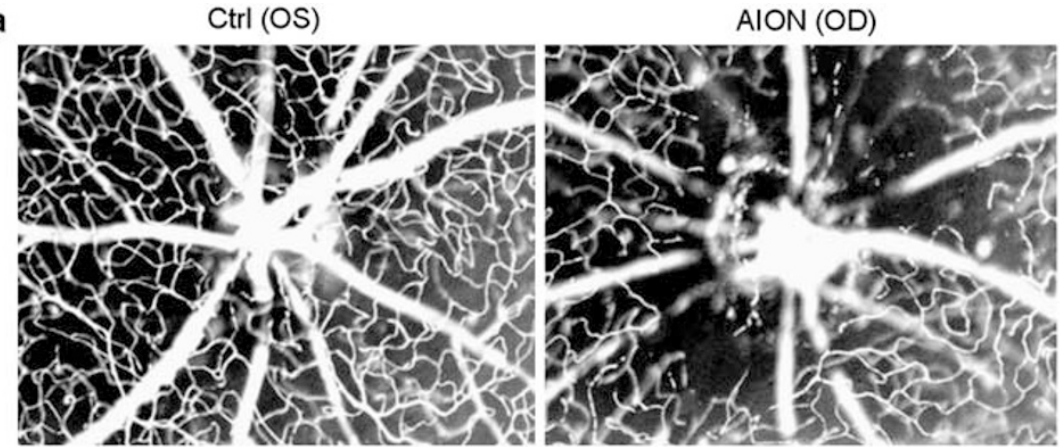

b
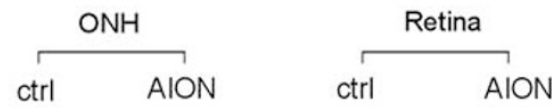

$\alpha-B$ crystallin

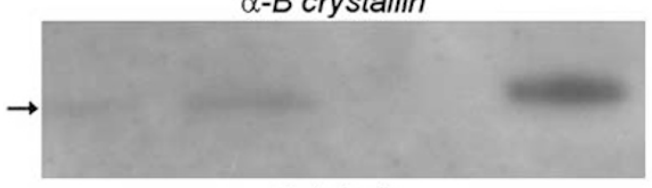

$\beta$-tubulin

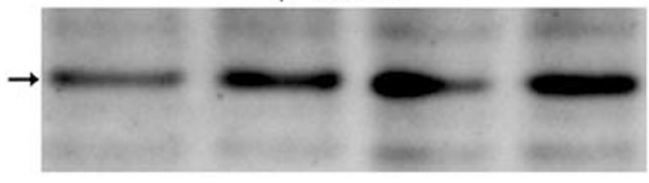

C

$\alpha \mathrm{BC} / \beta$-tubulin

$\mathrm{ONH}$ Retina

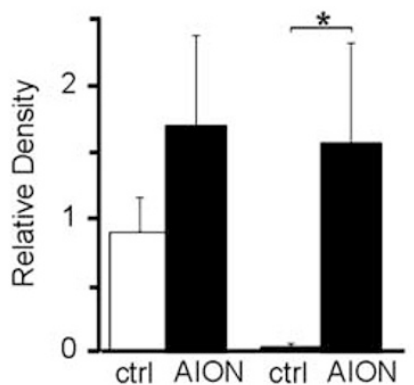

Figure 1 Experimental AION and early upregulation of $\alpha$ BC. (a) Retinal angiography following experimental AION showed prominent loss of peri-papillary small vessels OD. Ctrl: control, OD: right eye, OS: left eye. (b) Western blotting of control $v$ s AION ON head $(\mathrm{ONH})$ and retina 1 day following experimental AION showed increased $\alpha \mathrm{BC}$ protein expression in the AION eyes compared with control eyes. There was no significant change in the expression of class-III $\beta$-tubulin. (c) Quantification of western blot data ( $n=4$ experiments) revealed elevated $\alpha \mathrm{BC}$ expression compared with $\beta$-tubulin following AION. ${ }^{*} P<0.03$.
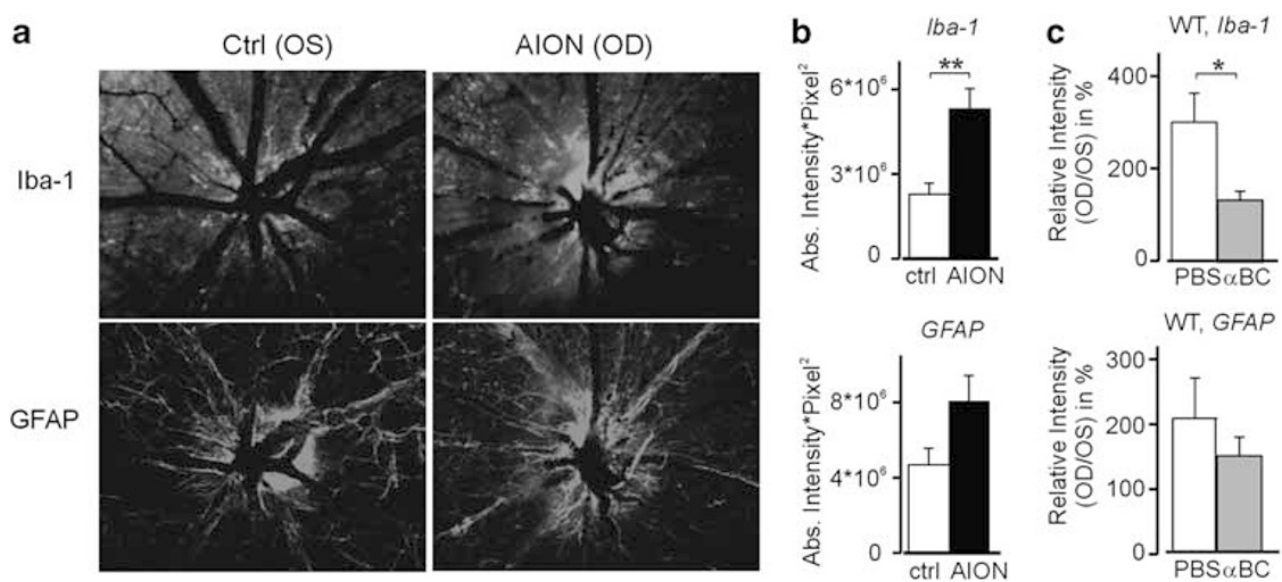

Figure 2 Acute post-AION microglial and astrocytic activation. (a) Increased ON head staining with Iba-1 and GFAP antibodies following AION. Eyes shown are the same as that of Figure 1a pictures. (b) Quantified, pooled data of PBS-treated mice showed increased Iba- 1 and GFAP antibody staining following AION. ${ }^{* *} P<0.002$. (c) Compared with PBS-treated animals, $\alpha$ BC-treated mice exhibited dampened post-AION microglial activation. There was a similar but not significant trend in the GFAP data. Relative intensity was calculated as the AION eyes normalized to paired control eyes. ${ }^{*} P<0.05$.

eyes (AION/control: $298 \pm 62 \%, n=8$ pairs of eyes, $P<0.002$ ) (Figures $2 \mathrm{a}$ and $\mathrm{b}$ ). This was consistent with post-AION microglial activation. The $\alpha \mathrm{BC}$ treatment effectively suppressed this microglial activation $(P<0.05)$ (Figure 2c), suggesting that $\alpha \mathrm{BC}$ decreased post-AION inflammation. Similar to Iba-1 antibody staining, the ON 
head GFAP antibody staining showed a trend of increased expression in the AION eyes compared with that of the control eyes ( $n=5$ pairs of eyes, $P=0.09$ ) (Figures $2 \mathrm{a}$ and $\mathrm{b}$ ). The $\alpha \mathrm{BC}$ treatment showed a trend of decreased GFAP upregulation (Figure 2c) (PBS: $202 \pm 62 \%, n=4$ pairs of eyes; $\alpha \mathrm{BC}: 147 \pm 29 \%, n=3$ pairs of eyes, $P>0.5$ ).

To monitor visual function, we recorded serial photopic flash VEP responses using electrodes implanted in the occipital lobes. Monocular light stimulation led to prominent contralateral occipital cortical responses, ${ }^{20}$ with increasing amplitudes and decreasing latencies of the $\mathrm{N}_{1}$ dominant peak in response to increasing luminance intensities (Figure 3a). We compared responses with $20 \mathrm{~cd} \mathrm{~s} / \mathrm{m}^{2}$, the brightest light stimuli used, between the $\alpha \mathrm{BC}$-treated and the control groups. Compared with baseline, at days 1-2 post-AION, there was a significant decrease of fVEP amplitudes in both saline- (baseline: $14.2 \pm 1.3 \mu \mathrm{V}$; days $1-2$ : $9.8 \pm 0.2 \mu \mathrm{V}$; $P=0.02, n=6$ ) and $\alpha \mathrm{BC}$-treated (baseline: $15.1 \pm 1.0 \mu \mathrm{V}$; days $1-2: 11.7 \pm 1.3 \mu \mathrm{V} ; P=0.04, n=6$ ) groups (Figure $3 \mathrm{~b}-\mathrm{c}$ ). There was no change in the latency of responses in both groups (saline: $P=0.7 ; \alpha \mathrm{BC}: P=0.3$ ) (Figure $3 \mathrm{~b}-\mathrm{c}$ ). These findings suggested that short-term $\alpha \mathrm{BC}$ treatment did not improve ON function early after experimental AION.

a
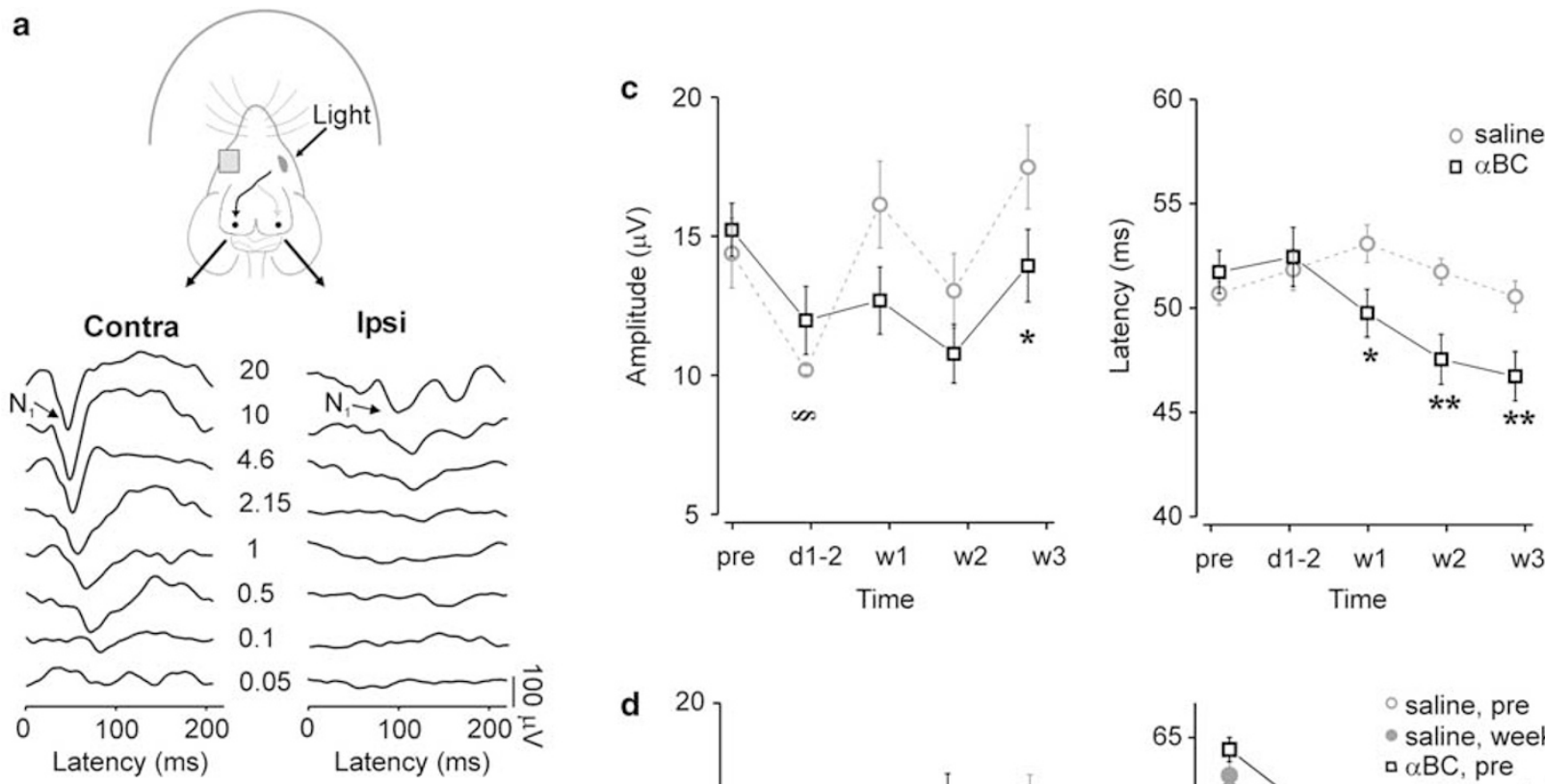

b
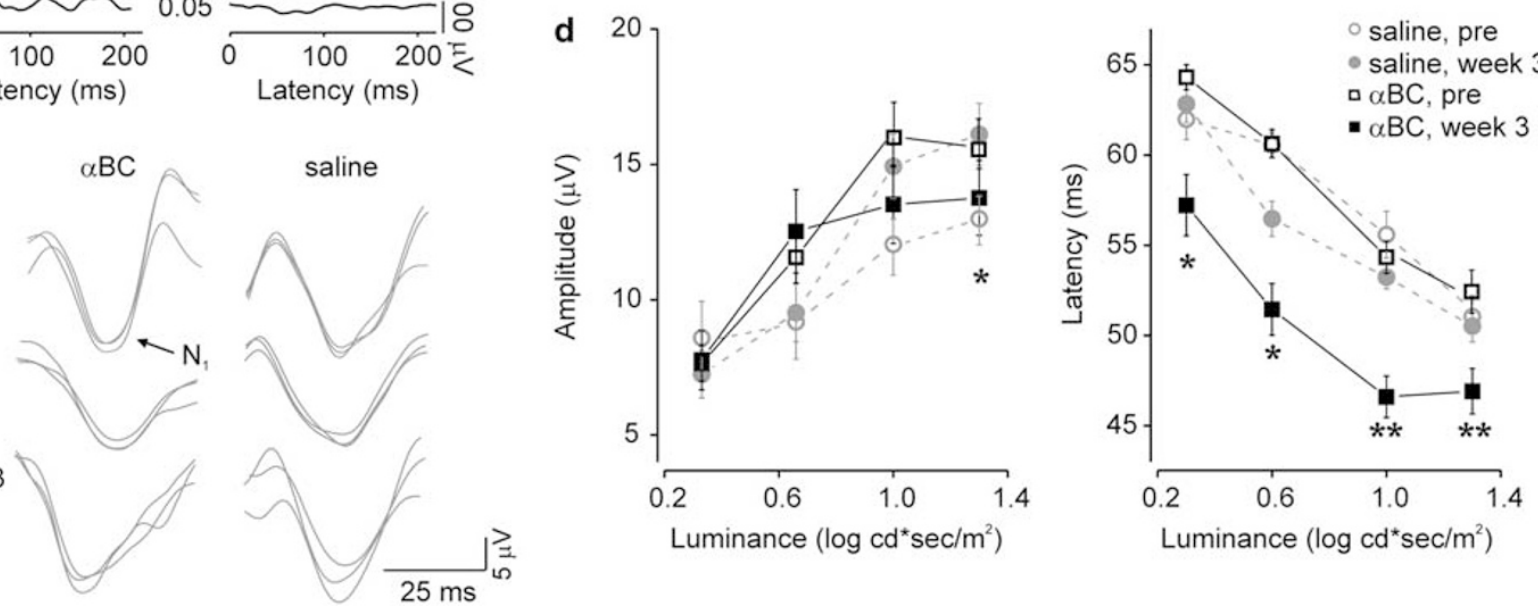

Figure 3 The $\alpha \mathrm{BC}$ treatment significantly improved the latencies of the intracranial photopic flash fVEPs. (a) Recording setup and visual evoked responses (stimuli in $\mathrm{cd} \mathrm{s} / \mathrm{m}^{2}$ ). Please note that an animal with relatively large fVEP response amplitudes were chosen to demonstrate luminance-dependent changes. (b) fVEPs from $\alpha \mathrm{BC}$-treated $(\alpha \mathrm{BC})$ and control (saline) groups. (c) Population fVEP data showed variability of fVEP amplitudes with no improvement in the $\alpha \mathrm{BC}$-treated group and significant acceleration of $\mathrm{fVEP}$ latencies from weeks 1 to 3 in the $\alpha \mathrm{BC}$-treated groups. ${ }^{\S} P<0.05$ (within each treatment group), ${ }^{*} P<0.05$, ${ }^{* *} P<0.002$ (saline $v s \alpha \mathrm{BC}$ groups). (d) Luminance intensity series at baseline $v s$ at 3 weeks showed variability and no differences of fVEP amplitudes across different luminance intensities. In contrast, there was improvement of fVEP latencies across all luminance intensities in the $\alpha \mathrm{BC}$-treated group. Note $y$ axis labels for (c) and (d) do not start at $0 .{ }^{*} P<0.03,{ }^{* *} P<0.009$. 


\section{Long-term $\alpha B C$ treatment}

For long-term treatment, we injected adult mice intravenously with $\alpha \mathrm{BC}$ (10 $\mu \mathrm{g}$ per injection) every other day for 3 weeks. Control group was injected with saline alone, and injections were performed under masked condition. We used fVEP recording to monitor visual function weekly over 3 weeks. Compared with days 1-2, at week 1, the fVEP amplitudes in the control group improved back to baseline (compared with days 1-2, $P<0.001$; compared with baseline, $P=0.2$ ) whereas the $\alpha \mathrm{BC}$-treated group had persistently low amplitudes (compared with days 1-2, $P>0.5$; compared with baseline, $P=0.07$ ). There was variability in the fVEP amplitudes throughout the 3 weeks, with overall improvement in both control and $\alpha \mathrm{BC}$-treated groups at 3 weeks compared with immediately post-AION.

The most striking finding in the long-term $\alpha \mathrm{BC}$ experiment was the fVEP latencies, which typically showed much less variability compared with the fVEP amplitudes. Compared with days $1-2$, by week 1 , the $\alpha \mathrm{BC}$-treated group demonstrated decreased response latencies (baseline: $52.2 \pm 1.1 \mathrm{~ms}$; week 1: $50.1 \pm 1.2 \mathrm{~ms}$; $P<0.05, n=6)$ while the latencies in the saline-treated group actually increased (baseline: $51.1 \pm 0.6 \mathrm{~ms}$; week 1 : $53.7 \pm 0.9 \mathrm{~ms} ; P<0.05, n=6)$. This acceleration of fVEP latencies in the $\alpha \mathrm{BC}$-treated group continued at weeks 2 and 3 (Figure $3 c$, right). Comparing the two treatment groups, the latency improvement in the $\alpha \mathrm{BC}$-treated group was significant at week $1(P=0.02)$, week 2 $(P=0.002)$ and week $3(P=0.009)$. By the third week of $\alpha \mathrm{BC}$ treatment, fVEP latencies at $20 \mathrm{~cd} \mathrm{~s} / \mathrm{m}^{2}$ were faster by $5.2 \mathrm{~ms}$ compared with days $1-2$ (d1-2: $52.1 \pm 1.3 \mathrm{~ms}$, week 3: $46.9 \pm 1.3 \mathrm{~ms}, P=0.02)$, whereas the latencies in the saline-treated group remained unchanged $(P=0.3)$.

Compared with baseline, the improvement of fVEP latency in the $\alpha \mathrm{BC}$-treated group at week 3 was evident across multiple luminance intensities (Figure $3 \mathrm{~d}$, right) $\left(2.15 \mathrm{~cd} \mathrm{~s} / \mathrm{m}^{2}: P=0.01,4.6 \mathrm{~cd} \mathrm{~s} / \mathrm{m}^{2}: P=0.03,10 \mathrm{~cd} \mathrm{~s} / \mathrm{m}^{2}\right.$ : $\left.P=0.001,20 \mathrm{~cd} \mathrm{~s} / \mathrm{m}^{2}: P=0.009\right)$, unlike the changes in the amplitudes, which were more variable and showed smaller fVEP amplitudes at $20 \mathrm{~cd} \mathrm{~s} / \mathrm{m}^{2}$ in the $\alpha \mathrm{BC}$-treated group compared with the control group $(P<0.05)$ but no difference at $10 \mathrm{~cd} \mathrm{~s} / \mathrm{m}^{2}$ (Figure $3 \mathrm{~d}$, left). These findings supported a significant functional improvement of the speed but not the amplitude of the visually evoked responses during the 3-week $\alpha \mathrm{BC}$ treatment.

\section{$\alpha B C$ rescued $O N$ oligodendrocytes but not $R G C s$}

We looked to see if the fVEP improvement in the $\alpha \mathrm{BC}$-treated group correlated with greater RGC survival. A total of $52 \pm 5$ fields were counted for the AION and control eyes in both treatment groups. At week 3, there was a significant loss of RGC layer cells ${ }^{5,8}$ in the AION eyes compared with the controlled eyes in both the $\alpha \mathrm{BC}$-treated group and in the control group $(P<0.001$ between AION and controlled eyes for both treatment groups) and no difference between the 2 treatment groups (Figure 4).

As fVEP latency is affected by myelination and the number and function of the ON oligodendrocytes, we analyzed if long-term intravenous $\alpha \mathrm{BC}(10 \mu \mathrm{g}$, every other day) vs saline treatment differentially affected $\mathrm{ON}$ oligodendrocyte survival. This is particularly important since weeks 1-2, when the fVEP latency started to differ
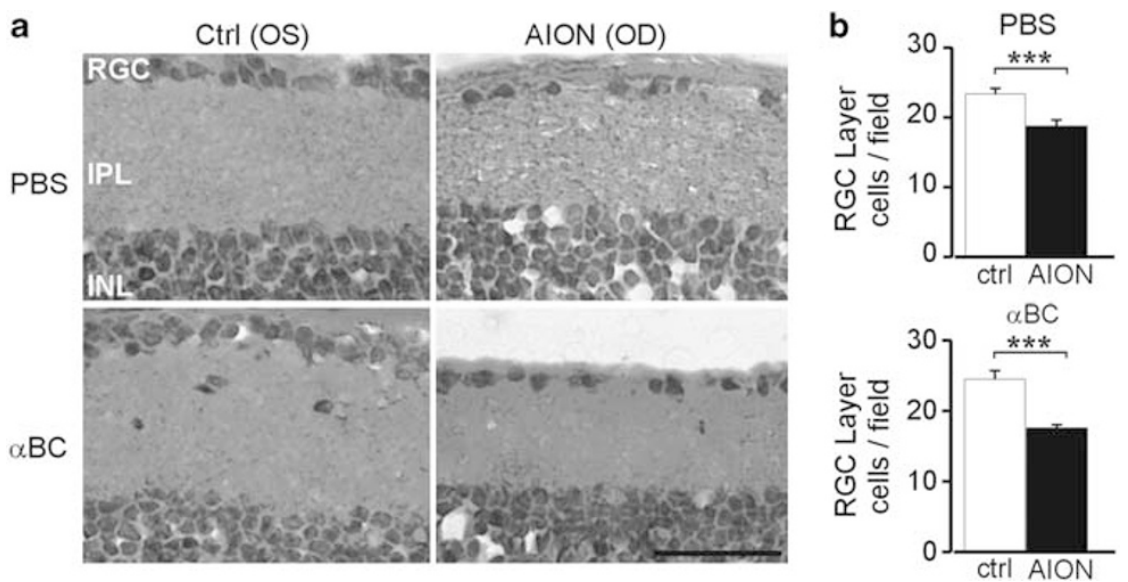

Figure 4 The $\alpha \mathrm{BC}$ treatment did not increase RGC survival. (a) Horizontal thin sections of mouse retina 3 weeks post-AION showed loss of cells in the RGC layer in both $\alpha$ BC-treated $(\alpha \mathrm{BC}$ ) and control (PBS) groups. (b) Quantification of RGC layer in AION and control eyes showed significant loss of RGC layer cells in the AION eyes in both treatment groups. A total of $52 \pm 5$ fields were counted for the AION and control eyes in both treatment groups. ${ }^{* * *} P<0.001$, within group. Scale bar: $0.05 \mathrm{~mm}$. 

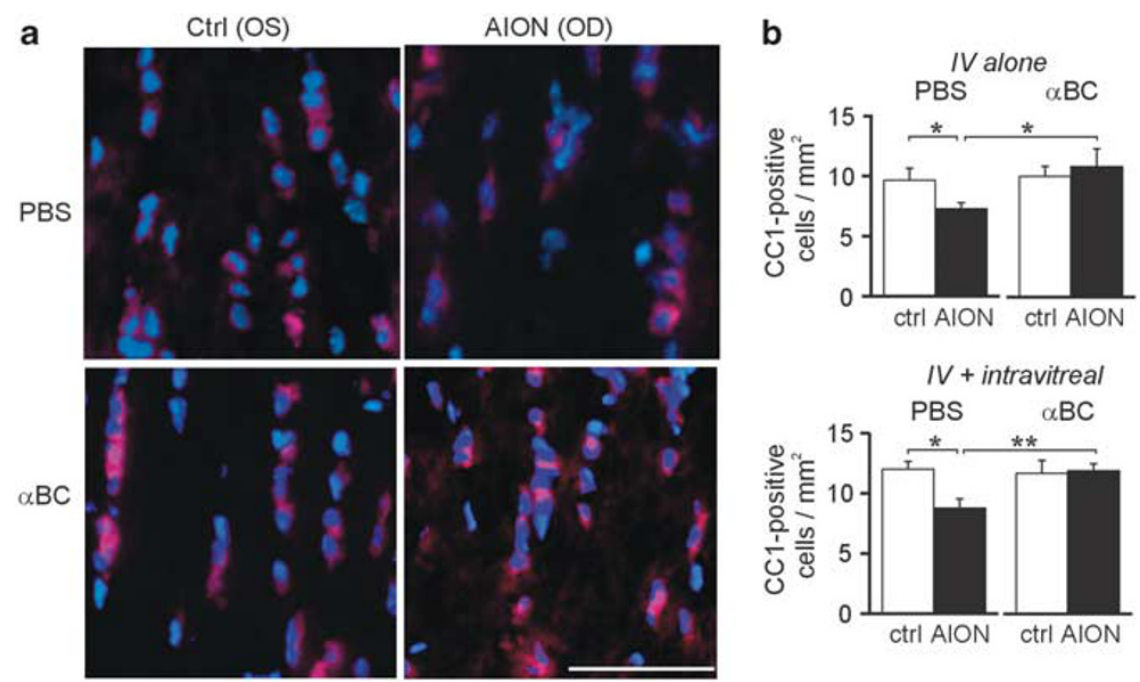

Figure 5 The $\alpha \mathrm{BC}$ treatment salvaged the ON oligodendrocytes. (a) Thin, horizontal sections of ONs at day 9 stained with antibody against CC1 (red, cytoplasmic stain), a marker of oligodendrocytes, and DAPI (blue, nuclear stain) showed fewer CC1 ${ }^{+}, \mathrm{DAPI}^{+}$ oligodendrocytes in the AION, saline-treated (PBS) group compared with the AION, $\alpha \mathrm{BC}$-treated group $(\alpha \mathrm{BC})$. There was no difference between the control eyes (Ctrl). OD, right eye; OS, left eye. (b) Top: quantification of $\mathrm{CC}^{+}{ }^{+}, \mathrm{DAPI}^{+}$cells in all animals showed complete rescue of ON oligodendrocytes after intravenous (IV) $\alpha \mathrm{BC}$ treatment. Bottom: population data of $\mathrm{CC}^{+}{ }^{+} \mathrm{DAPI}^{+}$cells in the intravenous plus intravitreal $\alpha B C$ treatment group compared with the saline-treated group. There was significant rescue of oligodendrocytes in the $\alpha$ BC-treated group. ${ }^{*} P<0.05,{ }^{* *} P \leq 0.005$. Scale bar: $0.05 \mathrm{~mm}$.

between the $\alpha \mathrm{BC}$-treated group compared with the control group, is known to be a peak period of ON oligodendrocytes loss following AION. ${ }^{8}$ In case the intravenously delivered $\alpha \mathrm{BC}$ did not reach the $\mathrm{ON}$ head due to vascular compromise, we also looked at the effect of additional intravitreal $\alpha \mathrm{BC}$ injection, which should deliver sufficient $\alpha B C$ to the ON head. On day 9 after treatment, during time of peak post-AION

oligodendrocyte loss, ${ }^{8}$ we counted the number of cells that stained positive for CC1 and DAPI, which corresponded to the number of oligodendrocytes. In the intravenous saline-treated group, there was 25\% fewer oligodendrocytes in the post-AION ONs compared with the control ONs (control: $9.7 \pm 1$ cells $/ \mathrm{mm}^{2}$, AION: $7.3 \pm 0.5$ cells $/ \mathrm{mm}^{2}, n=6-14$ fields per condition, $P<0.03$ ) (Figure 5). In contrast, in the intravenous $\alpha \mathrm{BC}$ treated group, there was a complete rescue of the $\mathrm{ON}$ oligodendrocytes following AION (control: $10.0 \pm 0.9$ cells $/ \mathrm{mm}^{2}$, AION: $10.8 \pm 1.5$ cells $/ \mathrm{mm}^{2}, n=6-14$ fields per condition) and significant improvement compared with the intravenous saline group $(P=0.02)$.

A combination of intravenous and intravitreal $\alpha \mathrm{BC}$ treatment also resulted in complete rescue of the $\mathrm{ON}$ oligodendrocytes following AION and significant improvement compared with the saline-treated group ( $n=5-11$ fields per condition per group, $P=0.005)$. These data showed that $\alpha \mathrm{BC}$ treatment promoted survival of ON oligodendrocytes, which correlated in timing with the significant fVEP latency improvement at weeks 1-2.

\section{Discussion}

Experimental AION led to early ON head swelling, loss of peripapillary vessels, ${ }^{1,2}$ upregulation of micro- and macroglia, and decreased fVEP responses. ${ }^{25,26}$ The upregulation of $\alpha \mathrm{BC}$, a molecular chaperone important in retinal ischemia, experimental glaucoma, and central nervous system inflammatory disease, ${ }^{14-16,27}$ following experimental AION was consistent with its role as a endogenous protective mechanism. ${ }^{28-30}$ Consistent with this idea, early, 3-day intravenous $\alpha \mathrm{BC}$ treatment dampened post-AION microglial activation, although it did not impact fVEP responses on days 1-2. Three-week $\alpha \mathrm{BC}$ treatment, however, led to significant improvement of the latency of the fVEP responses as early as week 1 , which was maintained at weeks 2 and 3 . This acceleration of the visually evoked responses correlated with the rescue of the ON oligodendrocytes on day 9 but not survival of RGCs at week 3. Intravenous treatment was as efficacious as intravenous plus intravitreal treatment. Taken together, our data suggested an $\mathrm{ON}$ protective effect of $\alpha \mathrm{BC}$ following $\mathrm{ON}$ head ischemia by dampening inflammation and salvaging oligodendrocytes.

Expressed in the RGCs ${ }^{34}$ and ON glia, ${ }^{16} \alpha \mathrm{BC}$ is poised to have a role in optic neuropathies. The upregulation of $\alpha \mathrm{BC}$ immediately following $\mathrm{ON}$ head ischemia is consistent with its function as a small heat shock protein with both protective and anti-apoptotic actions. ${ }^{14-16}$ 
Historically known as the predominant structural proteins in the lens, the $\alpha$-crystallins have been shown to act as molecular chaperones like Hsp70 and $\mathrm{GroE}^{14}$ and $\alpha \mathrm{BC}$ protects against UVA-induced apoptosis in cultured human lens epithelium. An upregulation of $\alpha B C$ has also been seen in response to hypoxia in cultured human $\mathrm{ON}$ head astrocytes, ${ }^{35}$ oxidative stress in cultured trabecular meshwork, ${ }^{36}$ chemical hypoxia in retina, ${ }^{37}$ and oxidative stress in cultured retinal pigment epithelium. ${ }^{38}$ Furthermore, $\alpha \mathrm{BC}$ upregulation may prevent apoptosis from oxidative stress in photoreceptors in uveitis. ${ }^{39}$ In the absence of $\alpha \mathrm{BC}$, retinal pigment epithelium is more susceptible to $\mathrm{H}_{2} \mathrm{O}_{2}$-induced apoptosis ${ }^{37}$ and exhibits greater post-ischemic inflammatory changes at the $\mathrm{ON}$ head, supporting $\alpha B C^{\prime}$ s role as a negative regulator of inflammation (this report). In contrast, progressive RGC degeneration observed in the ocular hypertension rat model for glaucomatous neuropathy is associated with a temporary downregulation of $\alpha \mathrm{BC}$ after 2 weeks and recovery after 5 weeks, ${ }^{31-33}$ suggesting a transient negative effect of ocular hypertension on $\alpha \mathrm{BC}$ gene expression. $^{32}$

The improvement in fVEPs responses following $\alpha \mathrm{BC}$ treatment developed over 3 weeks, during a period of known axonal and oligodendrocyte loss. ${ }^{8}$ The rescue of ON oligodendrocytes but not RGCs following experimental AION suggested that $\alpha \mathrm{BC}$ acted by protecting the $\mathrm{ON}$ but not neurons, presumably associated with an increased ratio of oligodendrocytes to axons in the ON. Curiously, fVEP latencies in the $\alpha \mathrm{BC}$-treated group improved beyond that of the baseline, which may be related to the direct effects of $\alpha \mathrm{BC}$ or from $\alpha \mathrm{BC}$-facilitated developmental acceleration of VEP latency. ${ }^{40}$ Aside from the effects on $\mathrm{ON}$ oligodendrocytes, we cannot rule out that $\alpha \mathrm{BC}$ treatment may have led to the selective survival of faster conducting RGCs, such as the parasol cells, over those of the slower conducting cells, such as the midget cells, ${ }^{41}$ which could explain the decreased latencies observed in the $\alpha \mathrm{BC}$ treated mice. Although the melanopsin-containing RGCs are known to be more resistant to ischemia, they do not contribute to evoked responses in the occipital lobe and therefore cannot account for the VEP latency changes.

Interestingly, $\alpha \mathrm{BC}$ treatment significantly improved paralysis and reduced inflammation in the central nervous system in an animal model of multiple sclerosis, ${ }^{15}$ a disease, which is also associated with VEP prolongation due to axonal and oligodendrocyte dysfunction. Injection of $\alpha \mathrm{BC}$ intravitreally has also been shown to improve $\mathrm{ON}$ axon density following crush injury. ${ }^{42}$ Here we provided compelling histological and functional evidence for the potential therapeutic value of a novel treatment $\alpha \mathrm{BC}$ in AION.
Summary

What was known before

- The $\alpha \mathrm{B}$-crystallin is an important molecule in inflammation and ischemia and is upregulated following different brain and eye injury models.

- Intravitreal treatment with $\alpha \mathrm{B}$-crystallin improved optic nerve crush model.

- Treatment with $\alpha \mathrm{B}$-crystallin improved animal model of multiple sclerosis.

What this study adds

- The $\alpha \mathrm{B}$-crystallin is upregulated rapidly following experimental anterior ischemic optic neuropathy.

- Treatment with $\alpha \mathrm{B}$-crystllin enhanced optic nerve function as shown by improvement in the latency of visually evoked responses. This was done with serial intracranial visual evoked potentials.

- The $\alpha \mathrm{B}$-crystallin improved optic nerve function by complete rescue of optic nerve oligodendrocytes following experimental anterior ischemic optic neuropathy.

\section{Conflict of interest}

The authors declare no conflict of interest.

\section{Acknowledgements}

We are grateful for the generous help of Yi-Wen Chen, Raymond Sobel, Sawsan Youssef, Mauricio Vargas, Michel Brahic, Huynh Van, and Phil Huie. YJL was supported by K08 NS044268 from the National Institute of Neurological Diseases and Stroke and the Career Award in Biomedical Sciences from the Burroughs Wellcome Foundation. LS and SSO were supported by the National MS Society. KK was supported by the Medical Scholars Program from Stanford University Medical School.

\section{References}

1 Arnold AC. Pathogenesis of nonarteritic anterior ischemic optic neuropathy. J Neuroophthalmol 2003; 23(2): 157-163.

2 Hayreh SS. Ischemic optic neuropathy. Prog Retin Eye Res 2009; 28(1): 34-62.

3 Potarazu SV. Ischemic optic neuropathy: models for mechanism of disease. Clin Neurosci 1997; 4(5): 264-269.

4 Levin LA, Danesh-Meyer HV. Hypothesis: a venous etiology for nonarteritic anterior ischemic optic neuropathy. Arch Ophthalmol 2008; 126(11): 1582-1585.

5 Bernstein SL, Guo Y, Kelman SE, Flower RW, Johnson MA. Functional and cellular responses in a novel rodent model of anterior ischemic optic neuropathy. Invest Ophthalmol Vis Sci 2003; 44(10): 4153-4162.

6 Chen CS, Johnson MA, Flower RA, Slater BJ, Miller NR, Bernstein SL. A primate model of nonarteritic anterior ischemic optic neuropathy. Invest Ophthalmol Vis Sci 2008; 49(7): 2985-2992.

7 Slater BJ, Mehrabian Z, Guo Y, Hunter A, Bernstein SL. Rodent anterior ischemic optic neuropathy (rAION) induces 
regional retinal ganglion cell apoptosis with a unique temporal pattern. Invest Ophthalmol Vis Sci 2008; 49(8): 3671-3676.

8 Goldenberg-Cohen N, Guo Y, Margolis F, Cohen Y, Miller NR, Bernstein SL. Oligodendrocyte dysfunction after induction of experimental anterior optic nerve ischemia. Invest Ophthalmol Vis Sci 2005; 46(8): 2716-2725.

9 Danylkova NO, Alcala SR, Pomeranz HD, McLoon LK. Neuroprotective effects of brimonidine treatment in a rodent model of ischemic optic neuropathy. Exp Eye Res 2007; 84(2): 293-301.

10 Bernstein SL, Guo Y, Slater BJ, Puche A, Kelman SE. Neuron stress and loss following rodent anterior ischemic optic neuropathy in double-reporter transgenic mice. Invest Ophthalmol Vis Sci 2007; 48(5): 2304-2310.

11 Danylkova NO, Pomeranz HD, Alcala SR, McLoon LK. Histological and morphometric evaluation of transient retinal and optic nerve ischemia in rat. Brain Res 2006; 1096: 20-29.

12 Munemasa Y, Kwong JM, Caprioli J, Piri N. The role of alphaA- and alphas in the survival of retinal ganglion cells after optic nerve axotomy. Invest Ophthalmol Vis Sci 2009; 50(8): 3869-3875.

13 Slavotinek AM, Biesecker LG. Unfolding the role of chaperones and chaperonins in human disease. Trends Genet 2001; 17(9): 528-535.

14 Andley UP. Crystallins in the eye: Function and pathology. Prog Retin Eye Res 2007; 26(1): 78-98.

15 Ousman SS, Tomooka BH, van Noort JM, Wawrousek EF, $\mathrm{O}^{\prime}$ onnor KC, Hafler DA et al. Protective and therapeutic role for alphaB-crystallin in autoimmune demyelination. Nature 2007; 448(7152): 474-479.

16 Oertel MF, May CA, Bloemendal H, Lutjen-Drecoll E. Alpha-B-crystallin expression in tissues derived from different species in different age groups. Ophthalmologica 2000; 214(1): 13-23.

17 Bernstein SL, Koo JH, Slater BJ, Guo Y, Margolis FL. Analysis of optic nerve stroke by retinal Bex expression. Mol Vis 2006; 12: 147-155.

18 Jain A, Blumenkranz MS, Paulus Y, Wiltberger MW, Andersen DE, Huie P et al. Effect of pulse duration on size and character of the lesion in retinal photocoagulation. Arch Ophthalmol 2008; 126(1): 78-85.

19 Porciatti V, Pizzorusso T, Maffei L. The visual physiology of the wild type mouse determined with pattern VEPs. Vision Res 1999; 39(18): 3071-3081.

20 Ridder III WH, Nusinowitz S. The visual evoked potential in the mouse - origins and response characteristics. Vision Res 2006; 46(6-7): 902-913.

21 Whiston EA, Sugi N, Kamradt MC, Heimer SR, Engelbert M Wawrousek EF et al. alphaB-crystallin protects retinal tissue during Staphylococcus aureus-induced endophthalmitis. Infect Imтии 2008; 76(4): 1781-1790.

22 Humphrey MF, Moore SR. Microglial responses to focal lesions of the rabbit retina: correlation with neural and macroglial reactions. Glia 1996; 16(4): 325-341.

23 Zhang C, Guo Y, Miller NR, Bernstein SL. Optic nerve infarction and post-ischemic inflammation in the rodent model of anterior ischemic optic neuropathy (rAION). Brain Res 2009; 1264: 67-75.

24 Watson BD, Dietrich WD, Busto R, Wachtel MS, Ginsberg MD. Induction of reproducible brain infarction by photochemically initiated thrombosis. Ann Neurol 1985; 17(5): 497-504.
25 Janaky M, Fulop Z, Palffy A, Benedek K, Benedek G. Electrophysiological findings in patients with nonarteritic anterior ischemic optic neuropathy. Clin Neurophysiol 2006; 117(5): 1158-1166.

26 Tormene AP, Doro D, Mantovani E, Fabris S, Moro F. Electrophysiological findings in anterior ischemic optic neuropathy. Metab Pediatr Syst Ophthalmol 1989; 12(1-3): 76-79.

27 Furuyoshi N, Furuyoshi M, May CA, Hayreh SS, Alm A, Lutjen-Drecoll E. Vascular and glial changes in the retrolaminar optic nerve in glaucomatous monkey eyes. Ophthalmologica 2000; 214(1): 24-32.

28 Sakaguchi H, Miyagi M, Darrow RM, Crabb JS, Hollyfield JG, Organisciak DT et al. Intense light exposure changes the crystallin content in retina. Exp Eye Res 2003; 76(1): 131-133.

29 Vazquez-Chona FR, Lu L, Williams RW, Geisert EE. Genomic loci modulating the retinal transcriptome in wound healing. Gene Regul Syst Bio 2007; 1: 327-348.

30 Yoshimura N, Kikuchi T, Kuroiwa S, Gaun S. Differential temporal and spatial expression of immediate early genes in retinal neurons after ischemia-reperfusion injury. Invest Ophthalmol Vis Sci 2003; 44(5): 2211-2220.

31 Ahmed F, Torrado M, Zinovieva RD, Senatorov VV, Wistow G, Tomarev SI. Gene expression profile of the rat eye iridocorneal angle: NEIBank expressed sequence tag analysis. Invest Ophthalmol Vis Sci 2004; 45(9): 3081-3090.

32 Piri N, Song M, Kwong JM, Caprioli J. Modulation of alpha and beta crystallin expression in rat retinas with ocular hypertension-induced ganglion cell degeneration. Brain Res 2007; 1141: 1-9.

33 Steele MR, Inman DM, Calkins DJ, Horner PJ, Vetter ML. Microarray analysis of retinal gene expression in the DBA/ 2J model of glaucoma. Invest Ophthalmol Vis Sci 2006; 47(3): 977-985.

34 Xi J, Farjo R, Yoshida S, Kern TS, Swaroop A, Andley UP. A comprehensive analysis of the expression of crystallins in mouse retina. Mol Vis 2003; 9: 410-419.

35 Yu AL, Fuchshofer R, Birke M, Priglinger SG, Eibl KH, Kampik A et al. Hypoxia/reoxygenation and TGF-beta increase alphaB-crystallin expression in human optic nerve head astrocytes. Exp Eye Res 2007; 84(4): 694-706.

36 Tamm ER, Russell P, Johnson DH, Piatigorsky J. Human and monkey trabecular meshwork accumulate alpha B-crystallin in response to heat shock and oxidative stress. Invest Ophthalmol Vis Sci 1996; 37(12): 2402-2413.

37 Yaung J, Jin M, Barron E, Spee C, Wawrousek EF, Kannan R et al. alpha-Crystallin distribution in retinal pigment epithelium and effect of gene knockouts on sensitivity to oxidative stress. Mol Vis 2007; 13: 566-577.

38 Alge CS, Priglinger SG, Neubauer AS, Kampik A, Zillig M, Bloemendal $\mathrm{H}$ et al. Retinal pigment epithelium is protected against apoptosis by alphaB-crystallin. Invest Ophthalmol Vis Sci 2002; 43(11): 3575-3582.

39 Saraswathy S, Nguyen AM, Rao NA. The Role of TLR4 in Photoreceptor \{alpha\}A crystallin upregulation during early EAU. Invest Ophthalmol Vis Sci 2010; 51(7): 3680-3686.

40 Heiduschka P, Schraermeyer U. Comparison of visual function in pigmented and albino rats by electroretinography and visual evoked potentials. Graefes Arch Clin Exp Ophthalmol 2008; 246(11): 1559-1573.

41 Sun W, Li N, He S. Large-scale morphological survey of mouse retinal ganglion cells. J Comp Neurol 2002; 451(2): 115-126.

42 Ying $X$, Zhang J, Wang Y, Wu N, Yew DT. Alpha-crystallin protected axons from optic nerve degeneration after crushing in rats. J Mol Neurosci 2008; 35(3): 253-258. 